$1967-09-15$

\title{
Very High Pressure Effects upon the EPR Spectrum of Ruby
}

H. Mark Nelson

D. B. Larson

J. H. Gardner

Follow this and additional works at: https://scholarsarchive.byu.edu/facpub

Part of the Astrophysics and Astronomy Commons, and the Physics Commons

\section{Original Publication Citation}

Nelson, H. M., D. B. Larson, and J. H. Gardner."Very High Pressure Effects upon the EPR Spectrum of Ruby." The Journal of Chemical Physics 47 (1967): 1994-1998.

\section{BYU ScholarsArchive Citation}

Nelson, H. Mark; Larson, D. B.; and Gardner, J. H., "Very High Pressure Effects upon the EPR Spectrum of Ruby" (1967). Faculty Publications. 804.

https://scholarsarchive.byu.edu/facpub/804 


\title{
Very High Pressure Effects upon the EPR Spectrum of Ruby*
}

\author{
H. M. Nelson, D. B. Larson, † and J. H. Gardner \\ Physics Department, Brigham Young University, Provo, Utah
}

(Received 29 March 1967)

\begin{abstract}
The variation in the electron paramagnetic resonance (EPR) spectrum of ruby as a function of pressure has been observed to beyond $70 \mathrm{kbar}$ for a magnetic field orientation parallel to the crystalline $c$ axis. The data can be interpreted in terms of the usual spin Hamiltonian: $x^{2}=g_{11} \beta H_{z} S_{z}-\frac{1}{2} \delta\left(S_{z}^{2}-\frac{1}{3} S^{2}\right)$, where the spectroscopic splitting factor $g_{\| 1}$ has the same value as at ambient pressures, but where the zero-field splitting $\delta$ increases in a linear fashion from $\sim 0.38$ to $\sim 0.43 \mathrm{~cm}^{-1}$. The experimental apparatus has been described previously, although important improvements in the pressure seal and in the pressure calibration have been made and are described in an Appendix.
\end{abstract}

\section{INTRODUCTION}

Electron paramagnetic resonance (EPR) would appear to be an ideal technique for probing the internal changes in a solid subjected to high pressure; the electrons are subjected to the very environment that it is desirable to study and they respond to changes in that environment very sensitively. Several workers ${ }^{1-3}$ have studied the pressure effects upon the EPR spectrum of solids, but they have done so in liquid pressure systems which have been limited in attainable pressure to below $10 \mathrm{kbar}$. This experiment uses Bridgman-type anvils $^{4}$ which makes possible the attainment of EPR results to pressures above $70 \mathrm{kbar}$. It must be recognized that the high-pressure EPR technique is still in the development stage; nevertheless these results for the pressure effects upon ruby up to $76 \mathrm{kbar}$ do indicate that experimental information can be elicited from EPR studies under the rigorous requirements of the experiment.

The theoretical problem of predicting high-pressure EPR effects, however, seems far from solution. Satisfactory theoretical models which would allow the computation of expected changes in an EPR spectrum under pressure are not available at present. The naive pointcharge model does not apply except perhaps in some ionic crystals, and phenomenological models like the spin Hamiltonian model make no attempt to relate the EPR spectrum to the parameters of the crystalline lattice. Because of the extreme complexity that a solid requires of a realistic fundamental model, progress toward it will undoubtedly require the accumulation of much experimental information.

\section{EXPERIMENTAL RESULTS}

Thirteen separate experimental runs are included in this report; for each run the external magnetic field was oriented along the $c$ axis of the trigonal ruby crystal;

\footnotetext{
* Supported by the National Science Foundation.

† Present address: Lawrence Radiation Laboratory, Livermore, Calif.

I W. M. Walsh, Phys. Rev. 114, 1485 (1959); 122, 762 (1961).

2 D. F. Wait, Phys. Rev. 132, 601 (1963).

A. F. Clark, R. H. Sands, and C. K. Kikuchi, Tech. Rept. ORA Project 06029, 1964.

4 P. W. Bridgman, Proc. Am. Acad. Arts Sci. 81, 165 (1952).
}

and each run had an internal bismuth pressure gauge as described in the Appendix.

The results are shown in Figs. 1 and 2 where the spread of the values of $\delta$ and $g_{11}$ for the pressures of the $\mathrm{Bi}$ I-II transition and the Bi III-V transition is indicated, as calculated by the spin Hamiltonian ${ }^{5}$

$$
\mathfrak{H C}=g_{1} \beta H_{z} S_{z}-\frac{1}{2} \delta\left(S_{z}^{2}-\frac{1}{3} S^{2}\right)
$$

for the several experimental runs. The specific values of $\delta$ and $g_{11}$, run by run, are listed in Table I. The pressure variation of $\delta$ obtained by Clark, Sands, and Kikuchi ${ }^{3}$ is shown in Fig. 1 for comparison.

The $g_{11}$ value of ruby does not vary measurably with pressure (Fig. 2). The $g_{11}$ value is calculated from $h \nu=g_{\|} \beta H_{c}$ where $\nu$ and $H_{c}$ are the klystron frequency and the magnetic field strength, respectively, appropriate for the $m_{\mathrm{a}}=-\frac{1}{2} \rightarrow m_{\mathrm{s}}=\frac{1}{2}$ transition in ruby. The constant $g_{11}$-value is considered to be $g_{11}=1.982+0.014$, -0.001 .

The horizontal line drawn in Fig. 2 is for $g_{11}=1.982$. The fact that the spread of data does not intersect this line at $76 \mathrm{kbar}$ is thought to be due to a slight misorientation of the sample at that pressure. A discussion of errors is included in the following section. At room pressure the $g_{\|}$value was measured to be $g_{11}=1.982 \pm 0.001$.

The value of $\delta$ varies linearly with pressure within the accuracy of the measurements. The slope of the line is

$$
d \delta / d P=6.0 \pm 0.4\left(\times 10^{-4} \mathrm{~cm}^{-1} / \mathrm{kbar}\right) \text {. }
$$

This slope should be compared with that obtained by Clark $e t a l .^{3}$

i.e.,

$$
d \delta / d P=7.27\left(\times 10^{-4} \mathrm{~cm}^{-1} / \mathrm{kbar}\right),
$$

$$
(21.15 \mathrm{Mc} / \mathrm{sec} / \mathrm{katm}) \text {. }
$$

The values of $\delta$ were calculated using

$$
\delta=h \nu\left[\left(H_{l} / H_{c}\right)+1\right]=h v\left[\left(H_{u} / H_{c}\right)-1\right],
$$

where $H_{l}$ and $H_{u}$ are the magnetic field strengths for the low-field $m_{\mathrm{s}}=\frac{3}{2} \rightarrow m_{\mathrm{a}}=\frac{1}{2}$ transition and the highfield $m_{s}=\frac{1}{2} \rightarrow m_{s}=\frac{3}{2}$ transition, respectively, and $H_{0}$ is

E. O. Shulz-Dubois, Bell System Tech. J. 38, 271 (1959) 


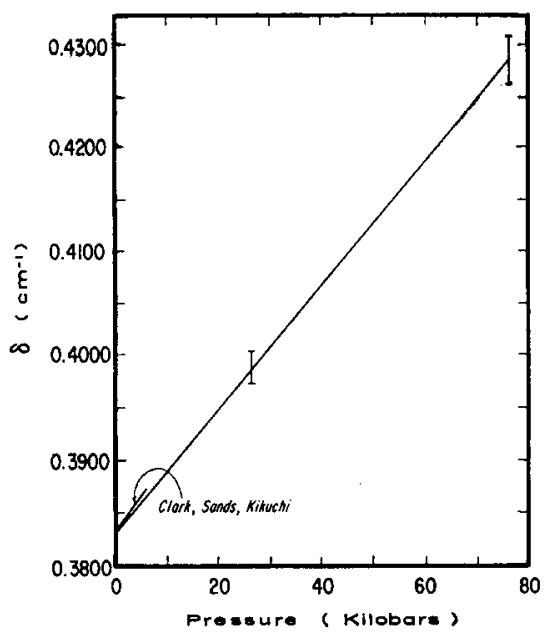

FIG. 1. Graph of zero-field splitting $\delta$ of ruby as a function of pressure. Bars indicate spread of data. Magnetic field parallel to crystalline $c$ axis. The results of Clark et al. (see Ref. 3 ) for $\delta$ as a function of pressure are shown for comparison.

the magnetic field strength for the central $m_{\mathrm{s}}=-\frac{1}{2}$ $\rightarrow m_{\mathrm{s}}=\frac{1}{2}$ transition.

The orientation dependences of $\delta$ and $g_{11}$ at room pressure are shown in Figs. 3 and 4 for the purpose of indicating the extent of error to be expected from a misorientation of the sample, assuming that a similar angular dependence exists at elevated pressures. Because $\delta$ calculated from $H_{l}$ (the lower curve in Fig. 3) is less sensitive to orientation than that calculated from $\boldsymbol{H}_{u}$, the former is plotted in Fig. 1.

Only the pressures of the Bi I-II transition and the Bi III-V transition are known with precision in our apparatus. The values of $\delta$ (and $\left.g_{11}\right)$ at these pressures were obtained by plotting $\delta$ (or $g_{11}$ ) vs oil pressure in the press and noting the oil pressure at which the transitions occurred. The EPR runs were not carried out at the pressures of the bismuth transitions themselves. It was felt that the pressure would be unstable there because of the changing volume of the bismuth and the very small size of the pressure cell.

Figure 5 is a tracing of two of the bismuth resistancevs-oil-pressure curves by means of which the bismuth transitions were detected. These curves are the worst

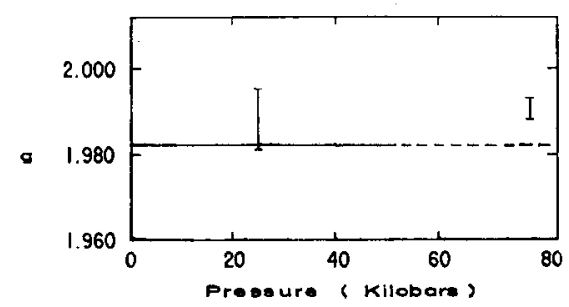

FIG. 2. Graph of spectroscopic splitting factor $g:$ of ruby (magnetic field parallel to crystalline $c$ axis) as a function of pressure. Bars indicate spread of data. The horizontal line indicates the assumed constant value of $g$ (see text for a discussion of errors).

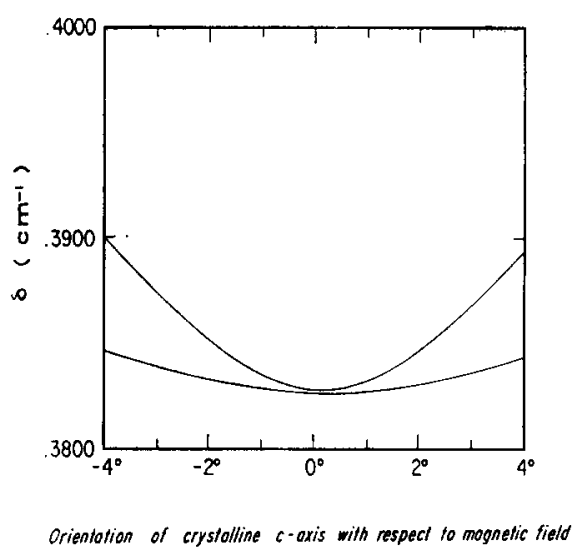

FIG. 3. Graph of zero-field splitting $\delta$ of ruby as a function of angle between magnetic field and crystalline $c$ axis. The upper curve was obtained using the data of the high field $m_{\mathrm{s}}=\frac{1}{2} \rightarrow m_{\mathrm{s}}=\frac{3}{2}$ transition and the lower curve was obtained using the data of the low field $m_{s}=\frac{3}{2} \rightarrow m_{8}=\frac{1}{2}$ transition. Data taken at room pressure.

and the best obtained by this technique. The tracing labeled "1 Sept." does not show a line for the Bí III-V transition because that transition occurred just after the recorder was disconnected for the resonance data to be taken. The spurious fluctuations in the measured resistance for the 1 September tracing are due to changing contact resistance at an initially poor connection. The resonance data corresponding to these two bismuth resistance-pressure curves are traced in Fig. 6. They reveal the line broadening which typically occurred in what was considered to be a successful run.

Some work was done at elevated pressures for two frequencies, one at either end of the X-band klystron tuning range $(\sim 8.5$ to $\sim 10.00 \mathrm{KMc} / \mathrm{sec})$ and for orientations of the magnetic field other than along the crystalline $c$ axis, but it seemed appropriate to include those results in a later report on a more detailed investigation of the relative validities at high pressure of the usual spin Hamiltonian and the more general symmetry Hamiltonian of Koster and Statz. ${ }^{6}$

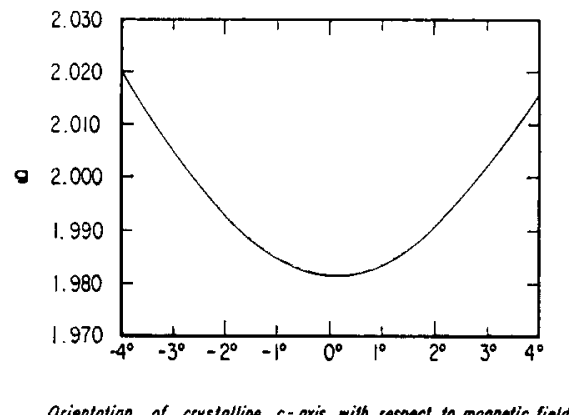

Fig. 4. Graph of spectroscopic splitting factor $g \|$ of ruby as a function of angle between magnetic field and crystalline $c$ axis. Data taken at room pressure.

6 G. F. Koster and H. Statz, Phys. Rev. 113, 445 (1959). 


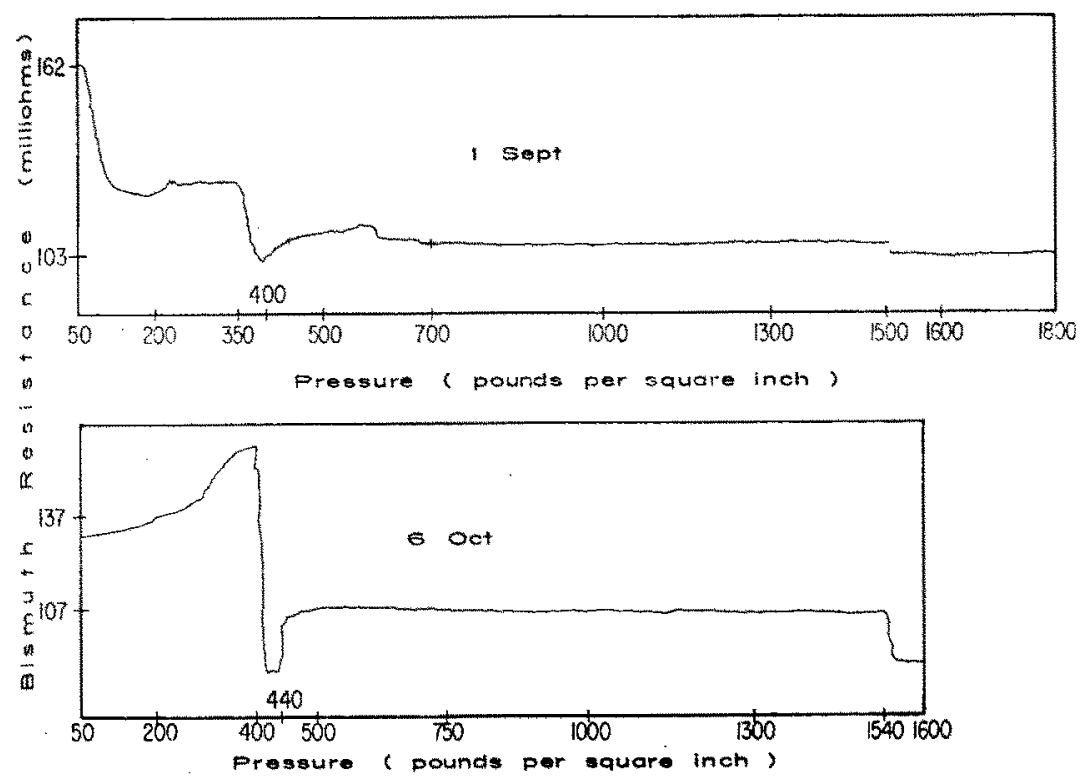

Frg. 5. Tracings of resistance-vspressure curves for bismuth in $E P R$ sample cell. The tracing labeled 1 Sept. does not show a line for the $\mathrm{Bi}$ III-V transition (at $1500 \mathrm{psi}$ ) because that transition occurred just after the recorder was disconnected for the resonance data to be taken.

No chemical analysis of the ruby was carried out, but the EPR linewidth indicated a mole concentration of $0.4 \%$ chromium. $^{7}$

\section{DISCUSSION}

Suspected sources of error in this experiment and their effects upon the zero-field splitting and $g_{11}$ value are tabulated in Table II. It should be noted that the scatter of results shown in Fig. 1 gives $\Delta \delta / \delta=0.008$ which exceeds any of the errors listed in the table except possibly the unknown error from sample distortion. That distortion perhaps arises because the pressure transmitting medium (indium) is not strictly hydro- static, but more likely the serious strains occur when the pressure seal is failing, allowing essentially uniaxial stresses to be placed upon the sample. There does not appear to be a direct correlation, however, between the data having the most line broadening and the data giving what is thought to be the worst values of $\delta$ (namely, the highest values for a given pressure).

At the present time there is no satisfactory theory of electron paramagnetic resonance in ruby which relates its spectrum to its crystalline parameters; consequently a theoretical prediction of the pressure effects upon the EPR spectrum of ruby is not available. Such a simple model as the point charge model is found to predict

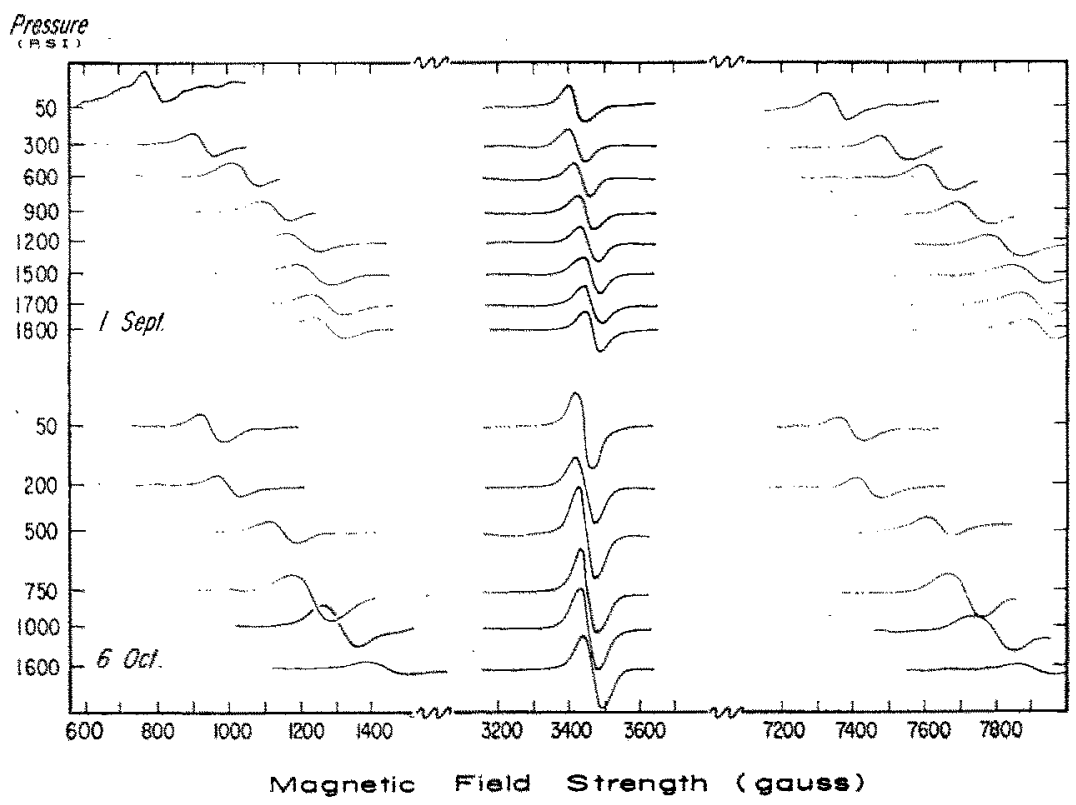

Fic. 6. Tracings of resonance data: Derivative of energy absorption vs magnetic field strength for the same runs whose pressure data are plotted in Fig. 5 .

7W. J. C. Grant and M. W. P. Strandberg, Phys. Rev. 135, A727 (1964), 
TABLE I. Values of spectroscopic splitting factor $g_{\|}$and zerofield splitting $\delta$ at 26 and $76 \mathrm{kbar}$.

\begin{tabular}{lccccc}
\hline & \multicolumn{2}{c}{$g_{\|}$} & & \multicolumn{2}{c}{$\delta\left(\mathrm{cm}^{-1}\right)$} \\
\cline { 5 - 6 } Date & $26 \mathrm{kbar}$ & $76 \mathrm{kbar}$ & & $26 \mathrm{kbar}$ & $76 \mathrm{kbar}$ \\
\hline 26 Aug. & 1.986 & & & 0.3974 & \\
29 Aug. & 1.987 & 1.986 & & 0.3975 & 0.4286 \\
30 Aug. & 1.985 & & & 0.3991 & \\
1 Sept. & 1.992 & 1.987 & & 0.3989 & 0.4283 \\
2 Sept. & 1.981 & & 0.3990 & \\
6 Oct. & 1.981 & & 0.3975 & 0.4318 \\
13 Oct. & 1.987 & & 0.3977 & \\
29 Oct. & 1.982 & & 0.3990 & \\
1 Nov. & 1.991 & & 0.4000 & \\
3 Nov. & 1.990 & & 0.4001 & \\
4 Nov. & 1.995 & & 0.4005 & 0.4262 \\
8 Nov. & 1.992 & 1.988 & & 0.3999 & 0.4302 \\
14 Nov. & 1.993 & & & & \\
\hline
\end{tabular}

even the wrong sign to the slope of the graph of zerofield splitting vs pressure, ${ }^{8}$ and models such as the spin Hamiltonian model or the more general Hamiltonian of Koster and $S t a t z^{6}$ are of the nature of phenomenological models where no attempt is made to relate spectra to Jattice parameters.

Macfarlane $e^{9}$ has made a quite successful calculation for room pressure of the zero field splitting of ruby using optical data to choose the parameters in the Hamiltonian. It seems feasible to carry out similar calculations at elevated pressures, where the optical data

TABLE II. Sources of experimental error and the effect of those errors upon the zero-field splitting $\delta$ and the spectroscopic splitting factor $g \|$.

\begin{tabular}{|c|c|c|c|}
\hline Source of error & $\Delta \delta / \delta$ & $\Delta g_{\|} / g_{\|}$ & Remarks \\
\hline Orientation & $<+0.003$ & $<+0.01$ & $\begin{array}{l}\delta \text { and } g_{\|} \text {have minima } \\
\text { for proper orienta- } \\
\text { tion; } \Delta \theta< \pm 3^{\circ} \text { by } g \\
\text { variation }\end{array}$ \\
\hline Magnetic field & $< \pm 0.002$ & $< \pm 0.003$ & $\underset{\text { measurement }}{ \pm 10 \mathrm{G} \text { by Hall probe }}$ \\
\hline $\begin{array}{l}\text { Klystron fre- } \\
\text { quency }\end{array}$ & $< \pm 10^{-6}$ & $< \pm 10^{-6}$ & $\begin{array}{l}\text { Electronic frequency } \\
\text { counter }\end{array}$ \\
\hline $\begin{array}{l}\text { Line broadening } \\
\text { and noise }\end{array}$ & $< \pm 0.002$ & $< \pm 0.001$ & $\begin{array}{l} \pm 10 \% \text { of peak-to-peak } \\
\text { width of derivative } \\
\text { trace; broadest line }= \\
110 \mathrm{G} \text { for } \mathrm{H}_{l} \text {, broad- } \\
\text { est line }=40 \mathrm{G} \text { for } H_{c}\end{array}$ \\
\hline $\begin{array}{l}\text { Pressure un- } \\
\text { certainty }\end{array}$ & $< \pm 0.002$ & $\cdots$ & $\begin{array}{l}\text { Uncertainty less than } \\
\text { the } 3 \text { kbar between } \\
\mathrm{Bi} \mathrm{I-II} \mathrm{transition} \mathrm{and} \\
\mathrm{Bi} \text { II-III transition, } \\
\text { slope of } \delta \text { vs } P=6 \times \\
10^{-4} \mathrm{~cm}^{-1} / \mathrm{kbar}\end{array}$ \\
\hline $\begin{array}{l}\text { Distortion of } \\
\text { sample crystal }\end{array}$ & Unknown & Unknown & \\
\hline
\end{tabular}

${ }^{8}$ D. B. Larson, Doctoral thesis, Brigham Young University, 1963 (unpublished).

${ }^{9}$ R. M. Macfarlane, J. Chem. Phys. 39, 3118 (1963). are available, to compare with the results of this experiment.

The procedure of Macfarlane ${ }^{9}$ (also used by Rimmer and Johnston ${ }^{10}$ ), may be criticized, however, because it cannot be justified on rigorous theoretical grounds. Löwdin ${ }^{11}$ has shown that the inner projection of the complete Hamiltonian matrix onto any subspace of finite order yields a contracted Hamiltonian matrix in which the matrix elements are energy dependent. Thus the effective Hamiltonian matrix should be sensibly different for each optical level used by these authors. Inasmuch as they ignore this energy dependence, the goodness of the fit, (which is only moderate), has only empirical significance. By contrast, for the case of the microwave spectra, the concept of an effective Hamiltonian with energy independent matrix elements should be a reasonably valid one as the relevant energy states

Fig. 7. High-pressure Bridgman-type anvil showing arrangement for introd ucing bismuth pressure indicator into EPR sample cell.


are very close together and are widely separated from the other energy states of the system.

\section{ACKNOWLEDGMENTS}

The authors wish to acknowledge the contributions of H. S. Kim, W. R. Hurren, and W. Gudmundson to the experimental aspects of this report and to acknowledge the contributions of many clarifying discussions with Dr. E. G. Larson.

\section{APPENDIX}

Figure 7 illustrates the technique used to measure the pressure within the sample cell. A piece of bismuth ( $\sim 0.070$ in. $X \sim 0.020$ in. $X \sim 0.001$ in. $)$ is inserted into the modulating circuit ${ }^{12}$ as shown. A very small amount of indium used as solder makes the connection between

${ }^{10}$ D. E. Rimmer and D. F. Johnston, Proc. Roy. Soc. (London) 89, 943, 953 (1966)

11 P.-O. Löwdin, J. Mol. Spectry. 14, 112 (1964).

12 J. H. Gardner et al., Rev. Sci. Instr. 34, 1043 (1963). 


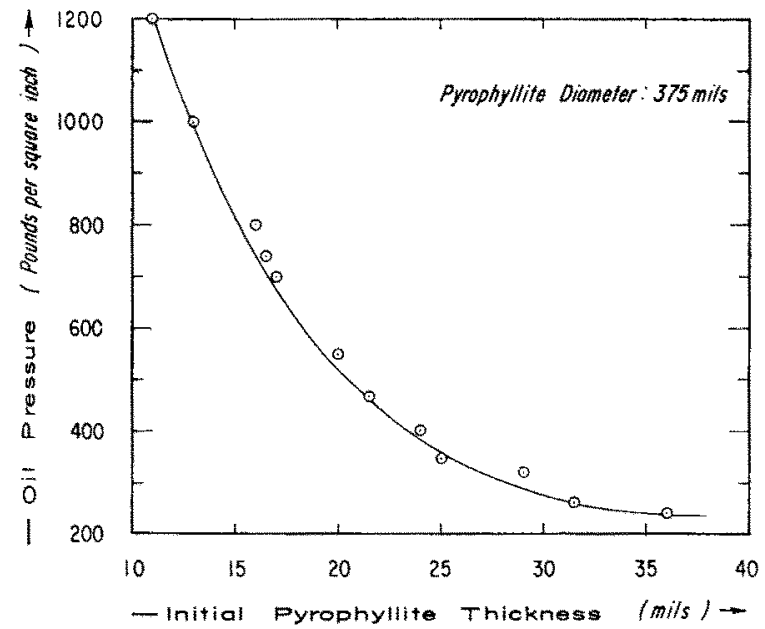

Fic. 8. Graph of oil pressure in the press required to achieve Bi I-II transition for various initial thicknesses of pyrophyllite pressure seal. The points plotted are experimental.

the bismuth and silver leads which are fired ${ }^{13}$ onto the aluminum oxide anvil. When the pressure transmitting medium is a conducting material (e.g. indium or lithium), a polyethylene bag, formed by extrusion in a punch (from 0.004-in-thick sheet) is used to insulate the conducting medium from the bismuth. The resistance of the bismuth gives an indication of the pressure in the sample cell, particularly at the abrupt changes in bismuth resistance which have become conventional pressure calibration points at $\sim 26 \mathrm{kbar}$ for the Bi I-II transition and at $\sim 76 \mathrm{kbar}$ for the Bi III-V transition. ${ }^{14,15}$ Apparently the actual pressure of these transitions depends upon several factors such as the shape of the bismuth sample, the type of pres-
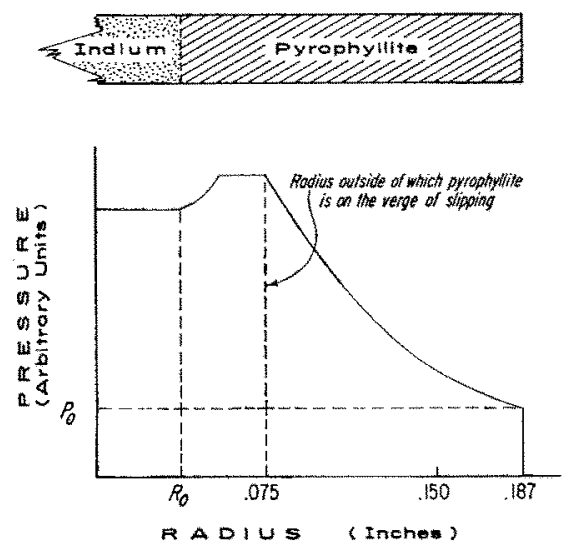

Frc. 9. Graph of pressure in the pyrophyllite pressure seal as a function of radius. The undeformed seal is shown to the same scale above the graph.

${ }^{13}$ The silver tape is available from the Vitta Corp., Wilton, Conn.

${ }^{14}$ R. N. Jeffery, J. D. Barnett, H. B. Vanfleet, and H. T. Hall, J. Appl. Phys. 37, 3172 (1966).

${ }^{15}$ G. C. Kennedy and P. N. Lamori, Publication No. 195 Institute of Geophysics, University of California. sure medium, whether the transition is approached from below or above, etc. The values: $26 \mathrm{kbar}$ for the Bi I-II transition and $76 \mathrm{kbar}$ for the Bi III-V transition, have been chosen as the apparent best values for the conditions of this experiment (flat, thin bismuth sample, solid pressure medium-indium, and increasing pressure at the transitions).

An analysis of the conditions for static equilibrium for a disk-shaped pressure seal under Bridgman-type anvils has been carried out by Jackson and Waxman.16 The results of a similar analysis have been applied to this system in an effort to optimize conditions and improve reliability. The relevant results are summarized ${ }^{17}$ in Figs. 8 and 9. For a washer-shaped pressure seal $\frac{3}{8}$ in. o.d. $\times 0.070$ in. i.d. the thickness for maximum pressure multiplication without blowing out seems to be about $0.030 \mathrm{in}$. A larger thickness allows the pyro-

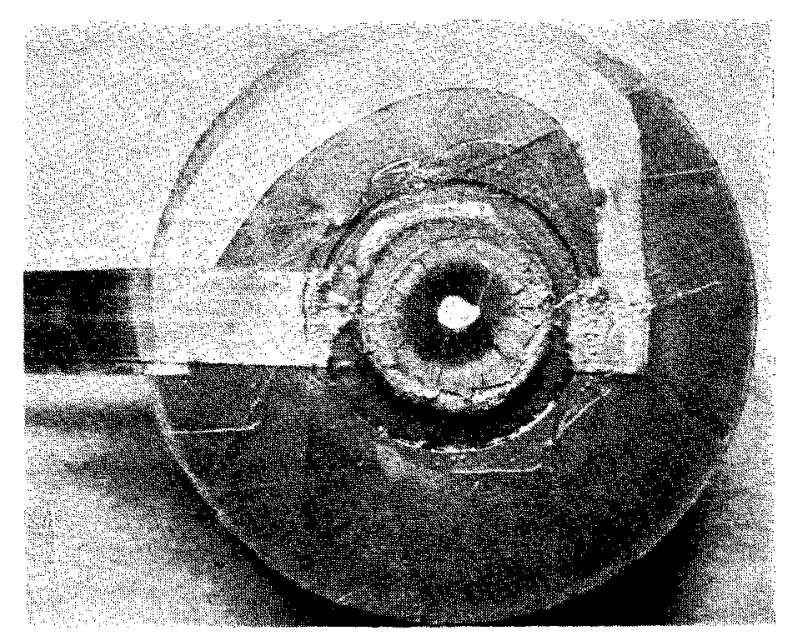

Frg. 10. Photograph of a pressure seal after use showing the penetration of the seal by the pressure transmitting medium.

phyllite to slip at the position of the hole, causing the pressure to be lost. Pressure seals made according to these specifications have proven to be about $80 \%$ reliable up to the pressure of the Bi I-II transition and about $20 \%$ reliable up to the pressure of the Bi III-V transition. The nature of the failure of the seal at these high pressures seems to confirm the information of Fig. 9. The pressure seal slips first along the silver modulation lead, letting the pressure transmitting medium channel out past the barrier formed by the most highly compressed pyrophyllite. The path taken by the escaped medium seems to be random, forming a meandering path through the remainder of the pyrophyllite, as shown in the photograph of Fig. 10.

${ }^{18}$ High Pressure Measurement, A. A. Giardini and E. C. Lloyd, Eds. (Butterworths Scientific Publications, Washington, D.C. 1962), p. 39 .

${ }_{17}$ The experimental points shown in Fig. 8 were obtained by W. Gudmundson and W. R. Hurren, Proc. Utah Acad. Sci. Arts and Letters 42, Part II, 328 (1965). 